\title{
Use of Activated Recombinant Factor VII in Severe Bleeding - Evidence for Efficacy and Safety in Trauma, Postpartum Hemorrhage, Cardiac Surgery, and Gastrointestinal Bleeding
}

\author{
Philip lau Victor Ong Wah Tze Tan Pei Lin Koh Mikael Hartman \\ Department of Surgery, National University Hospital, Singapore, Republic of Singapore
}

\section{Keywords}

Factor VII · Coagulation factors · Bleeding complication

\section{Summary}

Background: Uncontrolled bleeding continues to be a major cause of mortality in trauma, cardiac surgery, postpartum hemorrhage and liver failure. The aim of this paper is to assess the evidence supporting the efficacy of activated recombinant factor VII (rFVIla) administration in these settings. Methods: Electronic literature search. Results: Numerous retrospective trials have mostly shown a decrease in blood transfusion requirements with no increase in thromboembolic events (TEE), but major limitations in trial design make generalization difficult. In most retrospective reports rFVIla has been administered as a last-ditch attempt to control bleeding, when acidosis, hypothermia and coagulation factor depletion may not allow optimal rFVlla effect. Prospective randomized controlled trials have not shown any effect of rFVIla on mortality or TEE, although some have shown a reduction in RBC requirement. Conclusion: Stipulated transfusion protocols in prospective trials have reduced anticipated mortality among controls and make future trials for mortality effect unlikely in view of large sample size requirements. Establishment of these protocols and rapid hemostasis are likely to have greater benefits than administration of a single agent.

\section{Schlüsselwörter}

Factor VII · Gerinnungsfaktoren · Blutungskomplikation

\section{Zusammenfassung}

Hintergrund: Unkontrollierte Blutungen sind nach wie vor ein großes Problem während Trauma, Herzchirurgie, Post-partum-Hämorrhagie und Leberversagen. Das Ziel der vorliegenden Arbeit ist es, die Evidenz für die Effektivität der Verabreichung von aktiviertem rekombinantem Faktor VII (rFVIla) innerhalb dieser Settings zu bestimmen. Methoden: Elektronische Literatursuche. Ergebnisse: Eine Vielzahl retrospektiver Studien zeigte zumeist einen Rückgang des Bluttransfusionsbedarfs, ohne dass es zu einer Zunahme von thromboembolischen Ereignissen (TEE) kam; allerdings machten schwerwiegende Mängel beim Studiendesign eine Generalisierung schwierig. In den meisten retrospektiven Berichten wurde rFVIla als letzter verzweifelter Versuch eingesetzt, um die Blutung zu kontrollieren, wenn eine Azidose, eine Hypothermie und ein Gerinnungsfaktormangel möglicherweise einen optimalen rFVlla-Effekt verhindern. Prospektive randomisierte kontrollierte Studien zeigten keinen Effekt von rFVIla auf die Mortalität oder TEE, obwohl einige eine Reduzierung des Erythrozytenbedarfs nachweisen konnten. Schlussfolgerung: Festgeschriebene Transfusionsprotokolle in prospektiven Studien haben die erwartete Mortalität in der Kontrollgruppe reduziert und machen zukünftige Studien des Mortalitätseffekts im Hinblick auf die hohe Stichprobengröße unwahrscheinlich. Eine Etablierung derartiger Protokolle und eine schnelle Hämostase bewirken vermutlich einen größeren Benefit als die Verabreichung eines einzelnen Agens.

\section{KARGER \\ Fax +497614520714 \\ Information@Karger.de}

www.karger.com
(C) 2012 S. Karger GmbH, Freibur

$1660-3796 / 12 / 0392-0139 \$ 38.00 / 0$

Accessible online at:

www.karger.com/tm 


\section{Introduction}

Recombinant activated factor VII (rFVIIa; NovoSeven, Novo Nordisk, Bagsvaerd, Denmark) was initially developed for the treatment of hemophilia with coagulation factor inhibitors. It has since also been approved for the treatment of acquired hemophilia and other inherited bleeding diathesis such as Glanzmann thrombasthenia and factor VII deficiency. Its success in these clinical conditions as well as anecdotal reports of dramatic improvements when used as a 'last ditch' attempt in the treatment of coagulopathy has resulted in increased usage across various clinical scenarios. In the treatment of severe bleeding in non-hemophiliacs its off-label use persists despite the lack of high-level evidence for efficacy. The aim of this report is to review the evidence for safety and efficacy of rFVIIa usage for severe hemorrhage in the emergency setting. In particular we will review its use in severe trauma, gastrointestinal bleeding associated with liver failure, cardiac surgery and postpartum hemorrhage (PPH). Its use in bleeding prophylaxis and intracranial hemorrhage, while well reported, is beyond the scope of this review.

\section{Mode of Action}

rFVIIa appears to act via two separate pathways [1, 2]. The first is a tissue factor(TF)-dependent mechanism in which vascular damage leads to the TF availability. This leads to the formation of a TF-activated factor VII complex on activated platelets which in turn activates factor $\mathrm{X}$ and leads to the conversion of prothrombin to thrombin. At pharmacological levels, which is estimated at over 100 times the level of normal circulated FVII, rFVIIa has the additional effect of a TF-independent mechanism which activates factor $\mathrm{X}$ in the absence of TF. This pathway explains the ability of rFVIIa to bypass factor VIII and IX in hemophiliacs. rFVIIa at pharmacological levels may also down-regulate the fibrinolyic system through the production of thrombin-activatable fibrinolysis inhibitor (TAFI), a potentially pertinent action in severe trauma given the role of hyperfibrinolysis in acute coagulopathy of trauma (ACOT) [3].

Its mode of action [reviewed in 1, 2, 4] makes rFVIIa appealing as a systemic procoagulant in the treatment of refractory bleeding as TF availability and activated platelets are only available at active bleeding sites and should limit the danger thromboembolic events (TEE). Nevertheless the pharmacological doses involved and the background of elevated TEE risk in patients with severe trauma, $\mathrm{PPH}$ and cardiac and liver disease justifies arterial and venous thrombosis as the main focus of studies assessing the safety of rFVIIa use in these clinical settings. O'Connell et al. [5], reporting the incidence of TEEs in the Adverse Events Reporting System (AERS) of the US Food and Drug Administration (FDA), records 185 arterial and venous TEEs in 168 patients, most of whom received rFVIIa for ongoing bleeding. In the 102 reports in which a causality assessment for TEE was available, $81(79 \%)$ were thought to have a probable or possible causal relationship to rFVIIa. This is disconcertingly high rate of TEEs is worrisome especially as the AERS is an open reporting system that consists mainly of voluntary clinician reports and is likely to underreport actual incidence. Nevertheless the lack of a control group and information of baseline risk and concomitant medications hinders any assessment of causality to rFVIIa administration. Where controls are available, a more accurate evaluation of TEEs contributable to rFVIIa dosing is possible. In a review of TEEs in 35 randomized clinical trials for a wide spectrum of clinical scenarios in non-hemophiliacs, subjects administered with rFVIIa in various doses were noted to have in increased risk of arterial TEEs (5.5 vs $3.2 \%$; $p=0.003$ ), most commonly manifesting as coronary events. This increased risk was especially marked in subjects over 65 years of age [6].

The optimal dose and timing of rFVIIa in bleeding nonhemophiliacs has not been determined. Several in vitro and in vivo studies have demonstrated a decrease in rFVIIa activity with increasing acidosis at $\mathrm{pH} 7.2$ and below and a smaller effect of hypothermia [7]. A review of rFVIIa clearance in humans from pharmacokinetic profiling across a variety of clinical uses has also demonstrated rapid clearance with ongoing exsanguinations [8]. These studies support the earlier use of rFVIIa in the course of resuscitation of the bleeding patient and the need for repeated dosing, an approach which has shown to be beneficial in the military setting. Both these factors suggest that retrospective series which assess the use of rFVIIa and mostly consist of last-ditch attempts to secure hemostasis when all other conventional therapies have failed, may not represent optimal rFVIIa performance.

\section{rFVIla in Severe Trauma}

Trauma is the leading cause of death under the age of 45 years in developed countries. Of these, uncontrolled bleeding is the leading cause of preventable in-hospital death. Bleeding from trauma results from two mechanisms which require concurrent therapeutic strategies. 'Surgical' bleeding requires expedient control either by rapid surgical or interventional radiology techniques. In additional to rapid hemostasis, bleeding from trauma-related injuries also requires systemic replacement of hemostatic factors. The pathophysiology of traumarelated coagulopathy is multifactorial and has been extensively studied. A useful working model of a 'bloody vicious triad' described by Moore et al. [9] illustrates that a combination of the primary injury as well of secondary insults leads to dilution of coagulation products, acidosis, and hypothermia that further compromises clot formation leading to further blood loss. More recently an acute coagulopathy of trauma (ACOT) mediated via hyperfibrinolysis has been described 


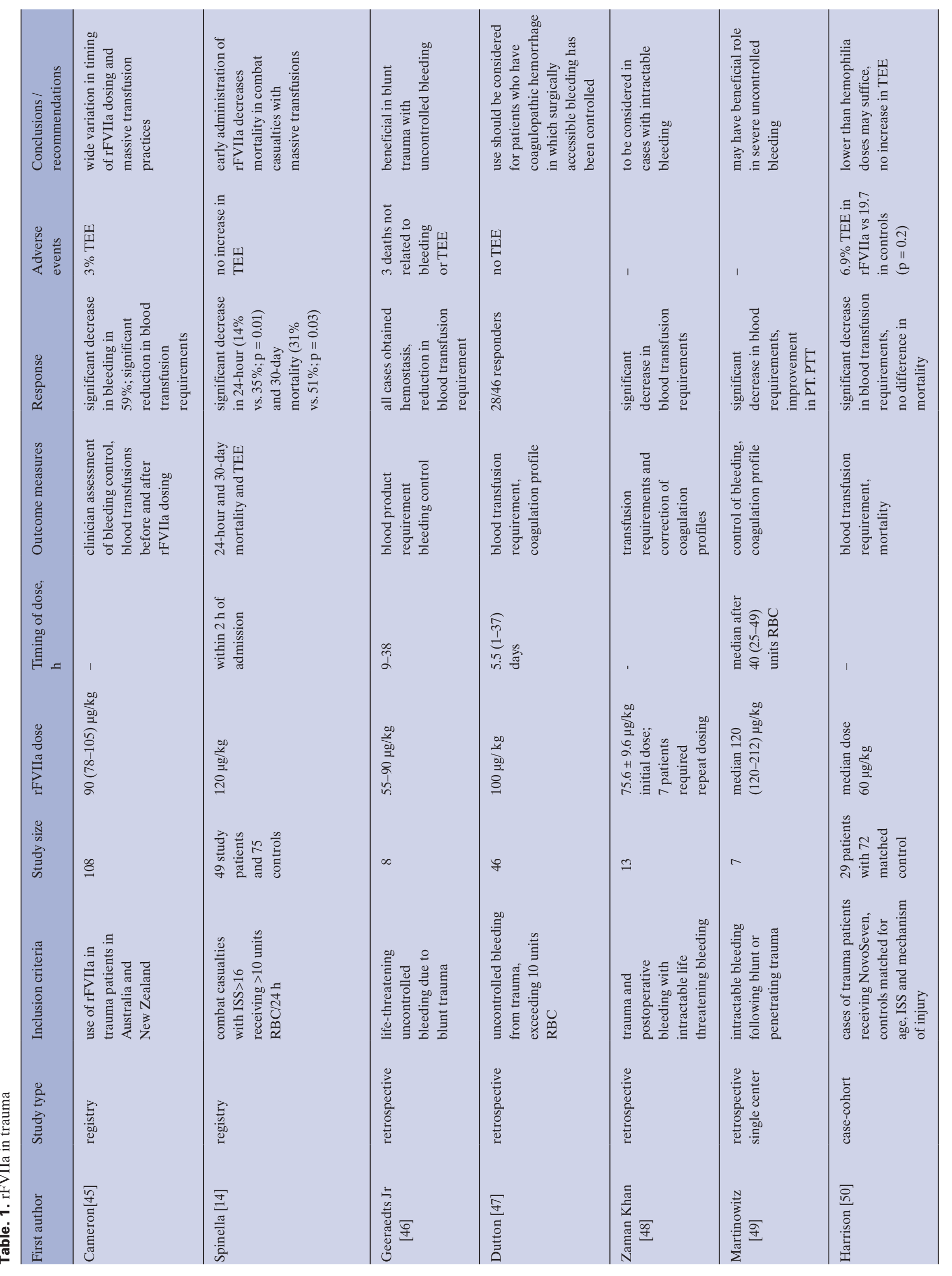


by Brohi et al. [10], resulting in early coagulation defects even before the onset of hypothermia and massive fluid resuscitation. Present in $25 \%$ of severely injured patients with acidosis even in the pre-hospital phase, it suggests the importance of early intervention with systemic procoagulants in this population of patients.

Since the first report of its use in the successful treatment of a severely injured young soldier [11], increasing off-label use in both in civilian and military settings have been reported. Since then numerous studies have reported on the efficacy of rFVIIa in the setting of trauma-associated coagulopathy. Given the relative rarity of exsanguinating hemorrhage in trauma, accounting for less than $5 \%$ of patients requiring blood transfusions, these studies have taken the form of retrospective case reports (table 1). A wide range of inclusion criteria, timing and dosing of rFVIIa, outcome measures, and statistical validity have been reported. Given the need for early and repeated doses of rFVIIa to maintain adequate levels in exsanguinating hemorrhage, whether all these trials represent its optimal use is debatable.

Inherent biases limit the validity of these retrospective series in assessing the efficacy and safety of rFVIIa in the trauma setting. There is a wide variation in the dose and timing of rFVIIa administration. Furthermore, the lack of a control group in the majority of studies does not permit the contribution of rFVIIa for TEE in a population inherently at risk for TEE. While decrease in blood transfusion requirements is consistently used as a surrogate to estimate bleeding control, this may also be as a result of correction of coagulopathy by standard methods, surgical or radiologic control, or even death.

Prospective clinical data on the use of rFVIIa in trauma are available from two randomized controlled trials. Boffard et al. [12] have reported on the single completed randomized controlled trial on rFVIIa in trauma-associated bleeding refractory of standard management. The study arm received $400 \mu \mathrm{g}$ of rFVIIa in three divided doses over a 2-hour period after the 4th RBC unit. A statistically significant reduction in RBC requirement was reported in the study arm. Criticisms of this trial include heterogeneity of treatment as 39 centers in 19 countries were included with no formalized transfusion protocols. Deaths prior to $48 \mathrm{~h}$ were not included in the analysis, and decreases in morbidity outcomes including multiple organ failure, ARDS, and sepsis were only conducted as a post-hoc analysis. No increase in TEE was noted.

The following CONTROL study [13] had planned to accrue 1,507 patients in order to demonstrate mortality benefit with rFVIIa administration, with a $30 \%$ anticipated mortality based on the previous trial and registry data. At the interim analysis following 573 randomized subjects, a mortality of only $11 \%$ and $18 \%$ in treatment arms for blunt and penetrating trauma patients, respectively, was recorded, with no significant difference to controls. The trial was terminated on grounds that it was unlikely to meet significant sample size for the primary endpoint for mortality benefit. A significant improvement from the previously reported trial was the institution of strict treatment protocols on ventilator weaning as well as blood product and fluid transfusions across all recruiting centers. It is likely that adherence to such evidence-based protocols may have a higher effect on survival than any intervention with a single agent such as rFVIIa.

While clinical data supporting a reduction in trauma mortality with rFVIIa is unlikely to be forthcoming, these randomized controlled trials do appear to suggest a comparable TEE rate to controls in this study population [14]. While the first randomized trial has been criticized for insufficient prospective observation for complications [13], in the CONTROL trial the $12 \%$ overall incidence of TEEs is higher than that reported by previous retrospective series as well as the $3 \%$ reported among similarly injured patients in the National Trauma Data Bank, with no increase in TEE in rFVIIa subjects versus controls.

\section{rFVlla in Bleeding Associated with Postpartum Hemorrhage}

Severe $\mathrm{PPH}$ is traditionally defined as estimated blood losses exceeding 11 after caesarian delivery or $500 \mathrm{ml}$ following vaginal delivery. Although encountered in less than $1 \%$ of deliveries, PPH persists as a significant contributor to maternal fatalities worldwide, contributing to about $25 \%$ of peripartum mortality $[15,16]$. Common causes are uterine atony, placental abnormalities, genital tract injury, and coagulation disorders. Accordingly, conventional treatment consists of fluid transfusion, oxytocin, misoprostol, and prostaglandin administrations, frequently followed by escalation to surgical maneuvers including ligation of ovarian and uterine arteries, B-Lynch suturing, bilateral internal iliac artery ligation, arterial embolization, and finally hysterectomy.

Studies reporting the use of rFVIIa have largely been based on retrospective reports (table 2) with large variations in timing and dosing of rFVIIa. Generally rFVIIa has been administered as a last-ditch attempt after liters of blood loss to prevent hysterectomy, although occasionally administration post hysterectomy for intractable nonsurgical bleeding has also been reported. In almost all cases, rFVIIa has been administered in the absence of any formal guidelines for its clinical indications, and reporting bias is likely. On the whole, however, a subjective reduction in bleeding and blood transfusion requirement has been observed in about two thirds of cases, suggesting its potential benefit in patients with PPH intractable to standard treatment.

The largest retrospective series is presented by the Australian and New Zealand Haemostasis Registry (ANZHR) which documented 110 patients in whom rFVIIa was administered for intractable PPH [17]. It appears that in most cases this was used as a 'last ditch' to avoid death from exsanguin- 


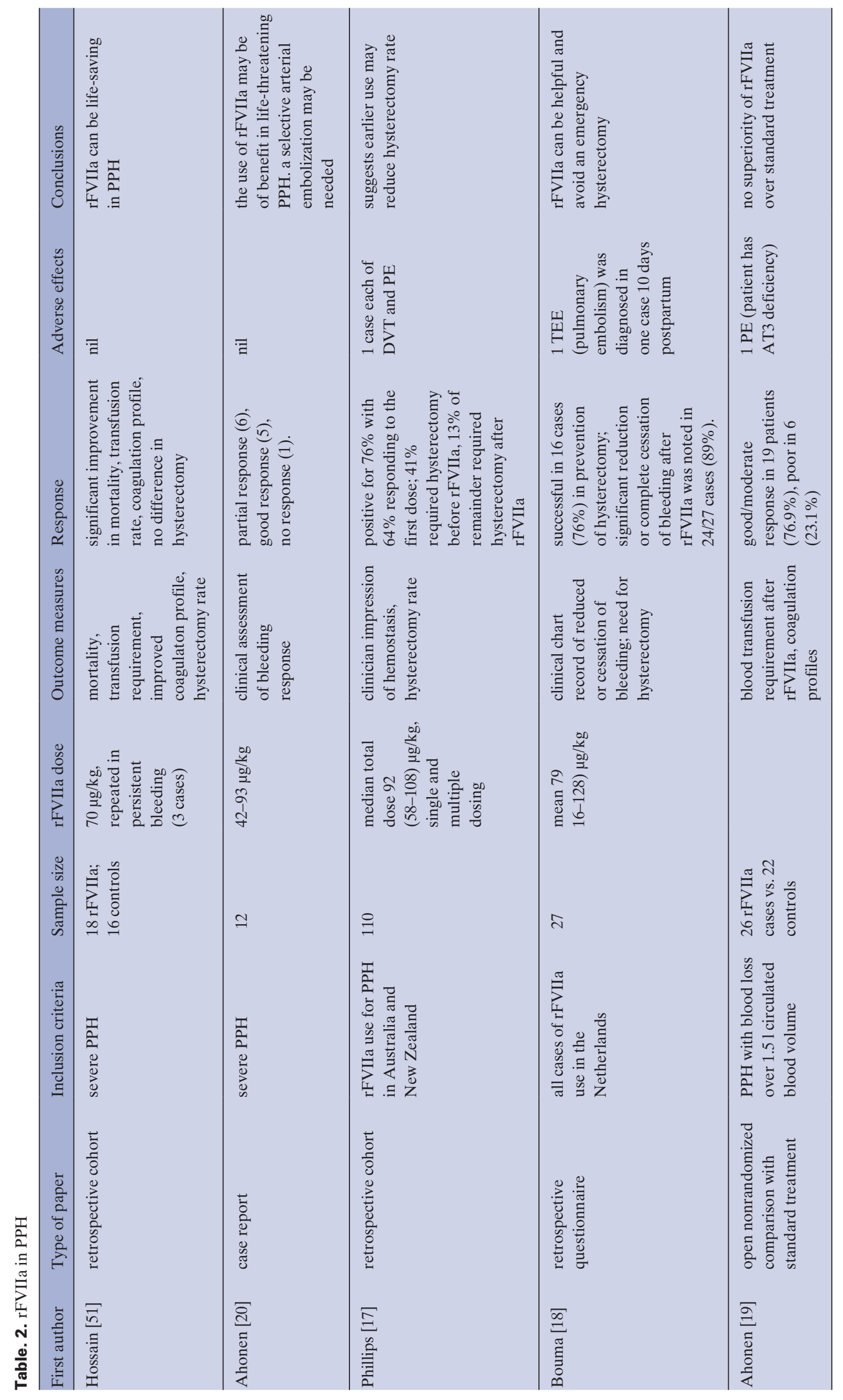


ation as over two thirds of patients had received over 15 units of RBCs before rFVIIa dosing and $41 \%$ had already undergone hysterectomy. Of the patients that had not undergone hysterectomy prior to rFVIIa dosing, this was required in $21 \%$ of cases, suggesting that earlier rFVIIa may improve uterine preservation. Bouma et al. [18], reporting on the experience of rFVIIa use for $\mathrm{PPH}$ in the Netherlands, found similar improvements in bleeding control. Again almost all patients had an estimated blood loss of over 31 before rFVIIa administration. Given how rFVIIa action may be compromised by acidosis, thrombocytopenia, hypothermia, and low fibrinogen levels attendant with this volume of blood loss [7], it is unlikely that these registry findings represent optimal rFVIIa performance. Despite an increased risk of TEE in this group of patients, these were rarely reported following rFVIIa use.

One prospective nonrandomized single center trial has been reported by Ahonen et al. [19]. Based on their previous experience in the use of rFVIIa in PPH [20], 26 patients were prospectively managed with rFVIIa using an institution protocol that included a rFVIIa dose of $90-120 \mu \mathrm{g} / \mathrm{kg}$, optimal platelet and fibrinogen levels, factor XIII support, and repeated rFVIIa dosing in refractory bleeding. When blood product and coagulation profiles were compared with 22 controls managed without rFVIIa over the same period, rFVIIa use was associated with poorer outcomes. The validity of these conclusions is debatable. rFVIIa was only considered when patients had lost at least 1.5 times their circulating blood volume. In the rFVIIa arm a mean of 9.91 of blood loss (range 4.2-19.7 1) had occurred before rFVIIa administration, which is greater than the mean total loss of 8.01 (range 5.0-19.0 l) in control patients. That poorer outcomes were seen in the rFVIIa arm is therefore not surprising.

No prospective randomized trials are available to conclusively assess the efficacy of rFVIIa in severe PPH. Given the rarity of PPH intractable to standard therapy, it is highly unlikely that this will ever be available and treatment may have to be determined on retrospective data. Given the high cost of rFVIIa, the inherent selection bias of these reports is inevitable as clinicians are likely to reserve and report the action of rFVIIa with a higher likelihood of response. In a review of available retrospective trials with 10 or more accrued subjects, Franchini et al. [21] observed that, with these reservations notwithstanding, over $85 \%$ of reported subjects had significant reduction in blood losses after a median administration of $81.5 \mu \mathrm{g} / \mathrm{kg}$ of rFVIIa, suggesting a clinically relevant role. For the treatment of refractory life-threatening $\mathrm{PPH}$, the authors recommended optimization of conditions for rFVIIa action, including correction of hypothermia, thrombocytopenia, acidosis and hypofibrinogenemia, and a $90 \mu \mathrm{g} / \mathrm{kg}$ loading dose, with repeated dosing if no clinical response is observed in $20 \mathrm{~min}$. Should there be no response after the second dose, a hysterectomy should be considered.

\section{rFVIla in Postcardiac Surgery Bleeding}

Intractable postcardiac surgery has been variously defined as ongoing bleeding that precludes sternal closure, surgical drains with blood loss exceeding $100 \mathrm{ml} / \mathrm{h}$, or the need for large-volume transfusions to maintain clinical stability, with or without repeat operations to exclude a surgical cause for ongoing blood losses. Constituting between 5 and $7 \%$ of the cardiac operations [22], these patients are at significantly higher risk for postoperative morbidity than the general cardiac surgery population, with mortality between 19 and 40\% [23].

Several studies have reported the effect of rFVIIa on postcardiac surgery, but their findings are difficult to translate into clinical recommendations. The majority are observational studies without controls. Subjects in these studies consist of a wide range of patients undergoing coronary bypass, valve surgery, aortic root surgery, or a combination of operations, making generalizations difficult. The larger of these studies have been summarized in table 3. Perhaps more than other clinical subgroups commonly associated with massive bleeding, cardiac patients are at especially high risk for the adverse event associated with systemic procoagulants such as rFVIIa, including myocardial infarction, ischemic stokes, and other TEE [24]. This and the absence of control groups in these studies have made the safety aspects and efficacy especially difficult to determine.

Consistent across almost all retrospective series is the reduction of blood loss after rFVIIa administration. This has occasionally been measured directly by surgical drains but most commonly by blood transfusion requirements pre and post dosing. The largest experience of rFVIIa usage in this setting comes from registry data. The ANZHR examined the efficacy of rFVIIa for intractable bleeding following cardiac surgery in 304 patients, $83 \%$ of whom received a single median dose of $93 \mu \mathrm{g} / \mathrm{kg}$ [25]. Hemostatic response was assessed by pre- and post-rFVIIa blood transfusion requirements and clinicians' assessment of response. Moderate to complete cessation of bleeding was assessed in $84 \%$ of patients with substantial reduction in blood transfusion requirements. TEE were noted in $4 \%$ of patients, which is consistent with previous reports of similarly complex cardiac surgery although no formal comparison was made. A later analysis of an expanded ANZHR study population by Willis et al. [26] found no difference in reduction in bleeding or 28-day mortality across different rFVIIa dosage groups from $<40$ to $>100 \mu \mathrm{g} / \mathrm{kg}$, suggesting that a lower dose may be adequate. A review of off-label rFVIIa use in 503 Canadian patients by Karkouti et al. [27] showed a reduction in blood transfusion requirements post dosing. Adverse events were compared with controls drawn from consecutive cases from the participating centers, with no significant difference in mortality or serious adverse events. In both Australian and Canadian registries rFVIIa administration was associated with a delay in the end of cardiopulmonary bypass - in the Canadian series the median delay was $280 \mathrm{~min}$, suggesting that 


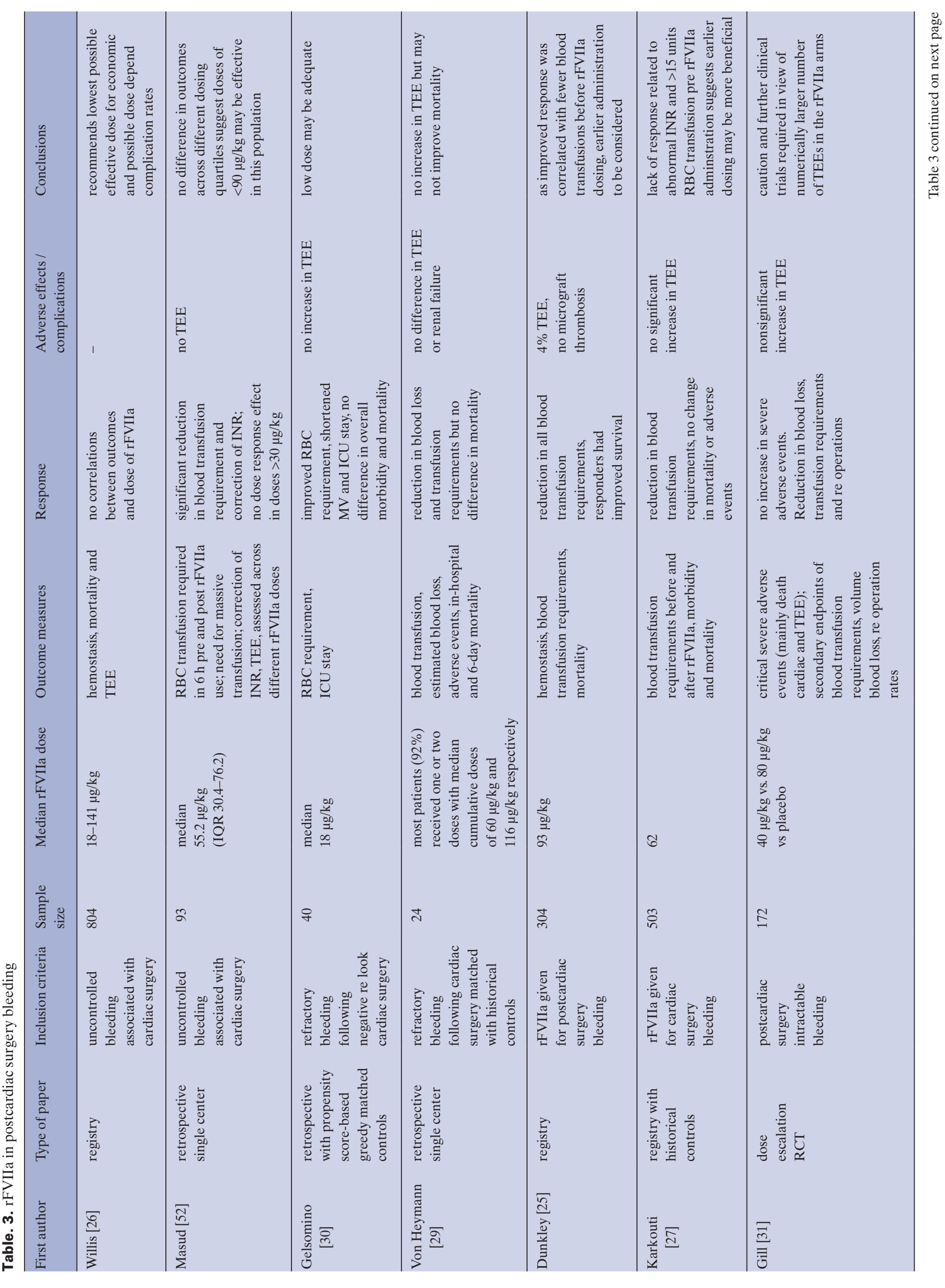




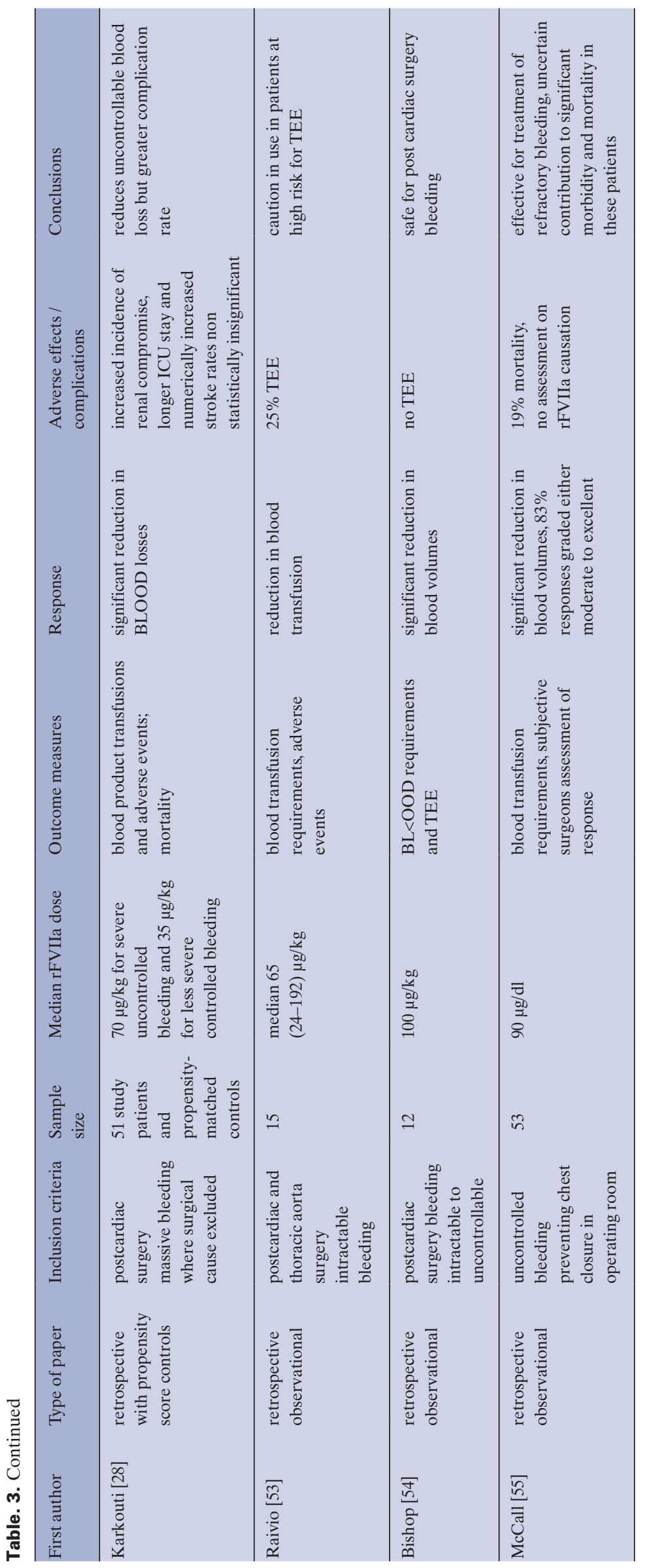

rFVIIa was truly a last-ditch attempt to achieve bleeding control. As the number of blood units transfused prior to rFVIIa administration was demonstrated as a predictor of lack of response to rFVIIa and mortality, both studies suggest earlier administration for maximum efficacy. Also notable in this large experience of off-label use is the absence of coronary micrograft thromboses.

Prior to the availability of adequately powered prospective randomized controlled trials, retrospective studies with some variation in approach have been used to determine the efficacy and safety of rFVIIa in this setting. Karkouti et al. [28] matched 51 postcardiac surgery patients with an equal number of controls selected by a propensity score for massive bleeding as determined by preoperative hemoglobin levels, weight, age, and gender. A significant reduction in blood product requirement was noted in the rFVIIa group although this was offset by an increased length of ICU stay and renal compromise, with a nonsignificant increase in the incidence of stroke. A possible confounding temporal effect may compromise this analysis, as the timing of rFVIIa administration was not taken in account in calculating its effect on blood transfusion requirements. To correct for this potential bias, von Heymann et al. [29] matched 24 postcardiac patients with an equal number of historical controls matching for volume of bleeding and other co-morbidities and the blood transfusion required up to and after the 24-hour postoperative mark, the median time in which rFVIIa was administered in the study group. Although blood loss and transfusions were reduced in the immediate period hours post rFVIIa infusion compared to controls, there was no difference in blood transfusions at the 24-hour mark, nor in the in hospitalization and mortality at 6 months. Reflecting the concerns for cost and TEE in this high-risk population, Gelsomino et al. [30] reported the effectiveness of a 1.2 $\mathrm{mg}$ rFVIIa dose (providing a median dose of $18 \mu \mathrm{g} / \mathrm{kg}$ ) in 40 cardiac patients with persistent blood loss compared to a control group with equal a priori probability of bleeding based on a propensity score analysis. Significant reductions in blood loss and transfusion requirements, length of mechanical ventilation, and hospital stay were noted.

Prospective data on the efficacy and safety of rFVIIa in the clinical setting of postcardiac surgery is limited to a phase II multicenter prospective randomized controlled trial reported by Gill et al. [31]. Patients admitted with severe bleeding following cardiac surgery in 30 participating centers were randomized in two separate cohorts to receive either rFVIIa $40 \mu \mathrm{g} / \mathrm{kg}$ or placebo or $80 \mu \mathrm{g} / \mathrm{kg}$ or placebo. Primary endpoints consisted of severe adverse events (SAEs) of interest, mainly myocardial and TEE complications, with secondary endpoints of volumes of blood requirement and re-operation rates. Unlike previous registry data from off-label rFVIIa use as a last-ditch effort to control bleeding, patients received trial drugs relatively early 


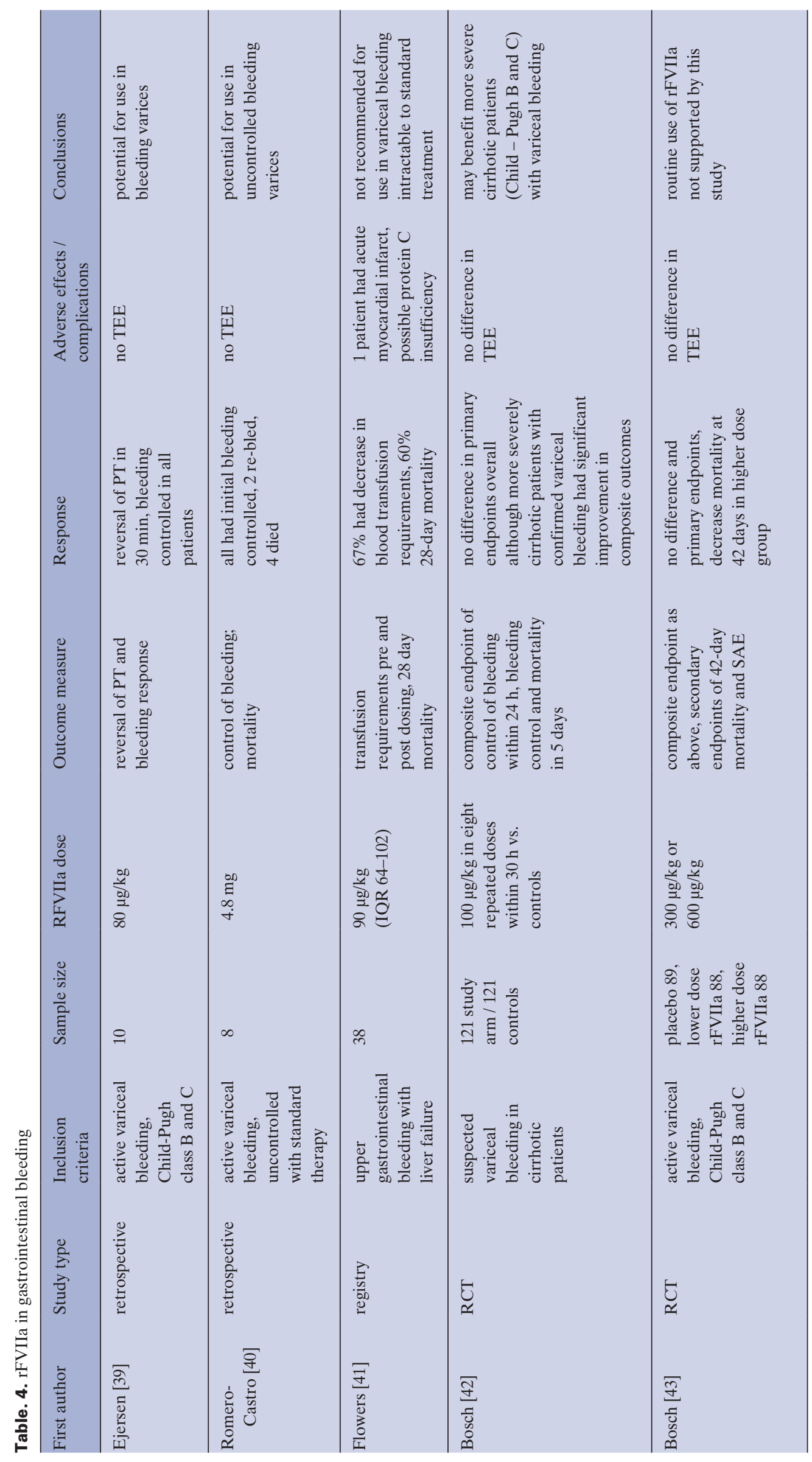


in their postoperative course, on average after just $2.8 \mathrm{~h}$ after arrival at the ICU, with control groups requiring a median of only $825 \mathrm{ml}$ of blood transfusion. Consistent with retrospective data, there was a significant decrease in blood transfusion requirement and estimated blood losses. However, patients receiving either dose had a numerically higher, statistically nonsignificant incidence of complications (4 cases of cerebral infarction vs. nil in placebo group, and 3 cases of other TEEs vs. nil in placebo group). rFVIIa given earlier in the course of postcardiac surgery appears to be efficacious, but the real correlation with TEE may require larger sample sizes

\section{rFVIla in Upper Gastrointestinal Bleeding and Liver Failure}

The liver plays a major role in maintaining hemostasis by the synthesis and regulation of most pro- and anti-thrombotic factors. Cirrhosis leads to a loss of this controlled regulation and can lead to both hyper- or hypocoagulable states. A bleeding diathesis is traditionally thought to arise from thrombocytopenia secondary to hypersplenism from portal hypertension, a loss of function of vitamin K-dependent clotting factors, and loss of platelet function from decreased thromboxane A2 [32, 33]. Conversely hypercoagulobility may ensue following an impaired fibrinolytic pathway secondary to decreased plasminogen and antiplasmin [34, 35].

Bleeding from esophageal and gastric varices is a common complication and cause of mortality of cirrhotic patients. Despite recent developments in the treatment of bleeding varices, there is a persistent $40 \%$ rate of re-bleeding, and mortality for initial bleeding and each re-bleeding episode is approximately $30 \%$ [36]. The potential of rFVIIa in treating cirrhosis-associated coagulopathy was first reported in studies which showed that single doses could reverse prolonged prothrombin time (PT) and correct international normalized ratios (INRs) [37, 38].

Two retrospective noncomparative trials assessed the efficacy of single doses of rFVIIa in patients with bleeding esophageal varices. Ejersen et al. [39] administered a single dose of $80 \mu \mathrm{g} / \mathrm{kg}$ to 10 Child-Pugh class B and C patients diagnosed with bleeding esophageal varices. Normalization of PT levels was obtained in $30 \mathrm{~min}$ and sustained over $7 \mathrm{~h}$. Bleeding in all cases was controlled with no increase in TEE. In a similar study Romero-Castro et al. [40] administered a single dose of $4.8 \mathrm{mg}$ of rFVIIa in 8 patients in whom active bleeding esophageal varices persisted despite treatment with endoscopy, vasopressors, and balloon tamponade. Hemostasis was obtained in all patients although 2 re-bleed within 1 week and 4 deaths occurred during the observation period. Flower et al. [41], reporting for the ANZHR, assessed the efficacy of rFVIIa in 38 cases of upper gastrointestinal bleeding associated with liver failure. Although $67 \%$ of patients were assessed to have reduced blood transfusions after rFVIIa ad- ministration, there was still a $60 \%$ 28-day mortality rate which was not different from that of non-responders. Notably as with other studies reporting off-label use, experience with rFVIIa in the ANZHR appeared to be mainly as a last-ditch effort, with $32 \%$ of patients already receiving more than 10 units of RBCs prior to rFVIIa administration. These patients appear to have a significant mortality regardless of whether bleeding is controlled or not.

Two randomized controlled trials by the European Study Group on rFVIIa on Upper GI Bleeding assess the feasibility of rFVIIa over and above standard treatment. The first was reported by Bosch at al. [42] in which patients with suspected variceal bleeding were randomized to either placebo or eight doses of $100 \mu \mathrm{g} / \mathrm{kg}$ of rFVIIa over a 30-hour period post randomization. The primary outcome measure was a composite endpoint consisting of control of bleeding within $24 \mathrm{~h}$ and rebleeding and death within 5 days of administration. No difference in the composite endpoint was noted in the rFVIIa compared to control patients. It should be noted that patients were often randomized on clinical diagnosis of variceal bleeding which was subsequently diagnosed in about two thirds of patients at endoscopy, during which about $45 \%$ had already shown no active signs of bleeding. On post-hoc analysis, patients with Child-Pugh class B and C cirrhosis with variceal bleeding receiving rFVIIa treatment had a significant decrease in failures in the composite endpoint, suggesting that rFVIIa may be of benefit in a more specific patient population with more severe liver disease. Of interest, the 5-day mortality of the intervention and control arms were $3 \%$ and $6 \%$, respectively, and thus much lower than the anticipated $30 \%$ seen in historical reports. This suggests that the strict adherence to optimal treatment protocols of vasoactive treatment, blood product transfusions, and therapeutic endoscopy in participating centers may have a larger effect on outcomes that the rFVIIa intervention. In a following study using the same endpoints with the additional secondary endpoints of adverse events and 42-day mortality, Bosch at al. [43] targeted 256 severely cirrhotic patients (Child-Pugh B and C) with endoscopically confirmed active variceal bleeding to receive either rFVIIa in divided doses of $300 \mu \mathrm{g} / \mathrm{kg}$ or $600 \mu \mathrm{g} / \mathrm{kg}$, or placebo within $1 \mathrm{~h}$ of diagnosis. Again no differences in the composite endpoints and 5-day mortality were noted; however, there was a significant decrease of 42-day mortality in the $600 \mu \mathrm{g} / \mathrm{kg}$ group compared to placebo, with death from bleeding significantly being reduced from 12 to $2 \%$. In neither of these RCTs a difference in TEE or SAEs between rFVIIa and placebo groups could be found (table 4).

\section{Conclusions}

Our review of the efficacy and safety of rFVIIa in emergency situations of massive blood loss suggests that with the present published evidence rFVIIa cannot be recommended as part of 
standard care for the treatment of massive bleeding in trauma, $\mathrm{PPH}$, cardiac surgery or gastrointestinal bleeding associated with liver failure. Concordant with the meta-analysis presented by Lin et al. [44], all RCTs carried out in these clinical situations have yielded a relative risk for mortality where $95 \%$ confidence intervals have included 1.0. It is unlikely that class I data for mortality benefit, which would require an estimated sample size of about 12,000 patients (based on an anticipated mortality in control groups of about $20 \%$ ) will ever be undertaken.

Aside from mortality outcomes, efficacy of rFVIIa has also been assessed with pooled blood transfusion requirements as a surrogate for hemostatic effect. Again the meta-analysis does not show conclusive evidence of a significant reduction in blood transfusion requirement. A consistent finding across randomized trials involving massive blood losses is the de- crease in anticipated mortality and blood requirements in studies in which a transfusion protocol was provided and enforced [13]. These would stipulate the administration of early RBCs, high RBC to plasma volumes, cryoprecipitate, fibrinogen, and other coagulation factor concentrates as well as expedient surgical and radiological hemostasis. It would appear that such comprehensive measures of optimizing bleeding control would have a larger efficacy in the treatment of these severely ill patients than can be expected by the intervention with a single agent.

\section{Disclosure Statement}

The authors did not provide a conflict of interest statement.

\section{References}

1 Hoffman M, Monroe DM 3rd: A cell-based model of hemostasis. Thromb Haemost 2001;85:958-965.

$\checkmark 2$ Hoffman M, Monroe DM 3rd: The action of highdose factor VIIa (fVIIa) in a cell-based model of hemostasis. Semin Hematol 2001;38:6-9.

>3 Brohi K, Cohen MJ, Ganter MT, Schultz MJ, Levi M, Mackersie RC, Pittet JF: Acute coagulopathy of trauma: hypoperfusion induces systemic anticoagulation and hyperfibrinolysis. J Trauma 2008; 64:1211-1217; discussion 1217.

4 Hedner U: Mechanism of action of factor VIIa in the treatment of coagulopathies. Semin Thromb Hemost 2006;32(suppl 1):77-85

$\checkmark 5$ O'Connell KA, Wood JJ, Wise RP, Lozier JN, Braun MM: Thromboembolic adverse events after use of recombinant human coagulation factor VIIa. JAMA 2006;295:293-298.

6 Levi M, Levy JH, Andersen HF, Truloff D: Safety of recombinant activated factor VII in randomized clinical trials. N Engl J Med 2010;363:1791-1800.

7 Viuff D, Lauritzen B, Pusateri AE, Andersen S, Rojkjaer R, Johansson PI: Effect of haemodilution, acidosis, and hypothermia on the activity of recombinant factor VIIa (NovoSeven). Br J Anaesth 2008;101:324-331.

8 Klitgaard T, Nielsen TG: Overview of the human pharmacokinetics of recombinant activated factor VII. Br J Clin Pharmacol 2008;65:3-11.

9 Moore EE, Burch JM, Franciose RJ, Offner PJ, Biffl WL: Staged physiologic restoration and damage control surgery. World J Surg 1998;22:11841190; discussion 1190-1191.

10 Brohi K, Singh J, Heron M, Coats T: Acute traumatic coagulopathy. J Trauma 2003;54:1127-1130.

11 Kenet G, Walden R, Eldad A, Martinowitz U: Treatment of traumatic bleeding with recombinant factor VIIa. Lancet 1999:354:1879.

12 Boffard KD, Riou B, Warren B, Choong PI, Rizoli S, Rossaint R, Axelsen M, Kluger Y: Recombinant factor VIIa as adjunctive therapy for bleeding control in severely injured trauma patients: two parallel randomized, placebo-controlled, double-blind clinical trials. J Trauma 2005;59:8-15; discussion 15-18.

13 Hauser CJ, Boffard K, Dutton R, Bernard GR, Croce MA, Holcomb JB, Leppaniemi A, Parr M, Vincent JL, Tortella BJ, Dimsits J, Bouillon B: Results of the control trial: efficacy and safety of recombinant activated factor VII in the management of refractory traumatic hemorrhage. J Trauma 2010;69:489-500.
14 Spinella PC, Perkins JG, McLaughlin DF, Niles SE, Grathwohl KW, Beekley AC, Salinas J, Mehta $S$, Wade CE, Holcomb JB: The effect of recombinant activated factor VII on mortality in combatrelated casualties with severe trauma and massive transfusion. J Trauma 2008;64:286-293; discussion 293-284.

15 Hazra S, Chilaka VN, Rajendran S, Konje JC: Massive postpartum haemorrhage as a cause of maternal morbidity in a large tertiary hospital. J Obstet Gynaecol 2004;24:519-520.

16 Khan KS, Wojdyla D, Say L, Gulmezoglu AM, Van Look PF: WHO analysis of causes of maternal death: a systematic review. Lancet 2006;367:10661074.

17 Phillips LE, McLintock C, Pollock W, Gatt S, Popham P, Jankelowitz G, Ogle R, Cameron PA: Recombinant activated factor VII in obstetric hemorrhage: experiences from the Australian and New Zealand Haemostasis Registry. Anesth Analg 2009;109:1908-1915.

18 Bouma LS, Bolte AC, van Geijn HP: Use of recombinant activated factor VII in massive postpartum haemorrhage. Eur J Obstet Gynecol Reprod Biol 2008;137:172-177.

19 Ahonen J, Jokela R, Korttila K: An open non-randomized study of recombinant activated factor VII in major postpartum haemorrhage. Acta Anaesthesiol Scand 2007;51:929-936.

20 Ahonen J, Jokela R: Recombinant factor VIIa for life-threatening post-partum haemorrhage. $\mathrm{Br} \mathrm{J}$ Anaesth 2005;94:592-595.

21 Franchini M, Franchi M, Bergamini V, Montagnana M, Salvagno GL, Targher G, Lippi G: The use of recombinant activated FVII in postpartum hemorrhage. Clin Obstet Gynecol 2010;53:219-227.

22 Marietta M, Facchini L, Pedrazzi P, Busani S, Torelli G: Pathophysiology of bleeding in surgery. Transplant Proc 2006;38:812-814.

23 Hein OV, Birnbaum J, Wernecke KD, Konertz W, Jain U, Spies C: Three-year survival after four major post-cardiac operative complications. Crit Care Med 2006;34:2729-2737.

24 Thomas GO, Dutton RP, Hemlock B, Stein DM, Hyder M, Shere-Wolfe R, Hess JR, Scalea TM: Thromboembolic complications associated with factor VIIa administration. J Trauma 2007;62:564569.
25 Dunkley S, Phillips L, McCall P, Brereton J, Lindeman R, Jankelowitz G, Cameron P: Recombinant activated factor VII in cardiac surgery: experience from the Australian and New Zealand Haemostasis Registry. Ann Thorac Surg 2008;85:836-844.

26 Willis C, Bird R, Mullany D, Cameron P, Phillips L: Use of rfVIIa for critical bleeding in cardiac surgery: dose variation and patient outcomes. Vox Sang 2010;98:531-537.

27 Karkouti K, Beattie WS, Arellano R, Aye T, Bussieres JS, Callum JL, Cheng D, Heinrich L, Kent B, Lee TW, MacAdams C, Mazer CD, Muirhead B, Rochon AG, Rubens FD, Sawchuk C, Wang S, Waters T, Wong BI, Yau TM: Comprehensive Canadian review of the off-label use of recombinant activated factor VII in cardiac surgery. Circulation 2008;118:331-338.

28 Karkouti K, Beattie WS, Wijeysundera DN, Yau TM, McCluskey SA, Ghannam M, Sutton D, van Rensburg A, Karski J: Recombinant factor VIIa for intractable blood loss after cardiac surgery: a propensity score-matched case-control analysis. Transfusion 2005;45:26-34.

29 von Heymann C, Redlich U, Jain U, Kastrup M, Schroeder T, Sander M, Grosse J, Ziemer S, Koscielny J, Konertz WF, Wernecke KD, Spies C: Recombinant activated factor VII for refractory bleeding after cardiac surgery - a retrospective analysis of safety and efficacy. Crit Care Med 2005; 33:2241-2246.

30 Gelsomino S, Lorusso R, Romagnoli S, Bevilacqua S, De Cicco G, Bille G, Stefano P, Gensini GF: Treatment of refractory bleeding after cardiac operations with low-dose recombinant activated factor VII (NovoSeven): a propensity score analysis. Eur J Cardiothorac Surg 2008;33:64-71.

31 Gill R, Herbertson M, Vuylsteke A, Olsen PS, von Heymann C, Mythen M, Sellke F, Booth F, Schmidt TA: Safety and efficacy of recombinant activated factor VII: a randomized placebo-controlled trial in the setting of bleeding after cardiac surgery. Circulation 2009;120:21-27.

32 Rios R, Sangro B, Herrero I, Quiroga J, Prieto J: The role of thrombopoietin in the thrombocytopenia of patients with liver cirrhosis. Am J Gastroenterol 2005;100:1311-1316. 
33 Senzolo M, Burra P, Cholongitas E, Burroughs AK: New insights into the coagulopathy of liver disease and liver transplantation. World J Gastroenterol 2006;12:7725-7736.

34 Hillenbrand P, Parbhoo SP, Jedrychowski A, Sherlock S: Significance of intravascular coagulation and fibrinolysis in acute hepatic failure. Gut 1974 15:83-88.

35 vanDeWater L, Carr JM, Aronson D, McDonagh $\mathrm{J}$ : Analysis of elevated fibrin(ogen) degradation product levels in patients with liver disease. Blood 1986;67:1468-1473.

36 Garcia-Tsao G: Current management of the complications of cirrhosis and portal hypertension: variceal hemorrhage, ascites, and spontaneous bacterial peritonitis. Gastroenterology 2001;120: 726-748.

-37 Bernstein DE, Jeffers L, Erhardtsen E, Reddy KR, Glazer S, Squiban P, Bech R, Hedner U, Schiff ER: Recombinant factor VIIa corrects prothrombin time in cirrhotic patients: a preliminary study. Gastroenterology 1997;113:1930-1937.

-38 Lisman T, Leebeek FW, Meijer K, Van Der Meer J, Nieuwenhuis HK, De Groot PG: Recombinan factor VIIa improves clot formation but not fibrolytic potential in patients with cirrhosis and during liver transplantation. Hepatology 2002;35:616-621.

-39 Ejlersen E, Melsen T, Ingerslev J, Andreasen RB Vilstrup H: Recombinant activated factor VII (rfVIIa) acutely normalizes prothrombin time in patients with cirrhosis during bleeding from oesophageal varices. Scand J Gastroenterol 2001; 36:1081-1085.

40 Romero-Castro R, Jimenez-Saenz M, PellicerBautista F, Gomez-Parra M, Arguelles Arias F, Guerrero-Aznar MD, Sendon-Perez A, HerreriasGutierrez JM: Recombinant-activated factor VII as hemostatic therapy in eight cases of severe hemorrhage from esophageal varices. Clin Gastroenterol Hepatol 2004;2:78-84.
41 Flower O, Phillips LE, Cameron P, Gunn K, Dunkley S, Watts A, Rajbhandari D: Recombinant activated factor VII in liver patients: a retrospective cohort study from Australia and New Zealand. Blood Coagul Fibrinolysis 2010;21:207-215.

42 Bosch J, Thabut D, Bendtsen F, D'Amico G, Albillos A, Gonzalez Abraldes J, Fabricius S, Erhardtsen E, de Franchis R: Recombinant factor VIIa for upper gastrointestinal bleeding in patients with cirrhosis: a randomized, double-blind trial. Gastroenterology 2004;127:1123-1130.

43 Bosch J, Thabut D, Albillos A, Carbonell N, Spicak J, Massard J, D'Amico G, Lebrec D, de Franchis R, Fabricius S, Cai Y, Bendtsen F: Recombinant factor VIIa for variceal bleeding in patients with advanced cirrhosis: a randomized, controlled trial. Hepatology 2008;47:1604-1614.

44 Lin Y, Stanworth S, Birchall J, Doree C, Hyde C: Use of recombinant factor VIIa for the prevention and treatment of bleeding in patients without hemophilia: a systematic review and meta-analysis. CMAJ 2011;183:E9-19.

45 Cameron P, Phillips L, Balogh Z, Joseph A, Pearce A, Parr M, Jankelowitz G: The use of recombinant activated factor VII in trauma patients: experience from the Australian and New Zealand Haemostasis Registry. Injury 2007;38:1030-1038.

46 Geeraedts LM Jr, Kamphuisen PW, Kaasjager HA, Verwiel JM, van Vugt AB, Frolke JP: The role of recombinant factor VIIa in the treatment of lifethreatening haemorrhage in blunt trauma. Injury 2005;36:495-500.

47 Dutton RPM, Hyder M, D'Angelo M, O'Connor J, Hess JR, Scalea TM: Factor VIIa for correction of traumatic coagulopathy. J Trauma 2004;57:709718; discussion 718-719.

48 Khan AZ, Parry JM, Crowley WF, McAllen K, Davis AT, Bonnell BW, Hoogeboom JE: Recombinant factor VIIa for the treatment of severe postoperative and traumatic hemorrhage. Am J Surg 2005;189:331-334.
49 Martinowitz U, Kenet G, Segal E, Luboshitz J, Lubetsky A, Ingerslev J, Lynn M: Recombinant activated factor VII for adjunctive hemorrhage control in trauma. J Trauma 2001;51:431-438; discussion 438-439.

50 Harrison TD, Laskosky J, Jazaeri O, Pasquale MD, Cipolle M: 'Low-dose' recombinant activated factor VII results in less blood and blood product use in traumatic hemorrhage. J Trauma 2005;59:150154.

51 Hossain N, Shamsi T, Haider S, Soomro N, Khan NH, Memon GU, Farzana T, Ansari S, Triche EW, Kuczynski E, Lockwood CJ, Paidas MJ: Use of recombinant activated factor VII for massive postpartum hemorrhage. Acta Obstet Gynecol Scand 2007:1-7.

-52 Masud F, Bostan F, Chi E, Pass SE, Samir H, Stuebing K, Liebl MG: Recombinant factor VIIa treatment of severe bleeding in cardiac surgery patients: a retrospective analysis of dosing, efficacy, and safety outcomes. J Cardiothorac Vasc Anesth 2009; 23:28-33.

53 Raivio P, Suojaranta-Ylinen R, Kuitunen AH: Recombinant factor VIIa in the treatment of postoperative hemorrhage after cardiac surgery. Ann Thorac Surg 2005;80:66-71.

54 Bishop CV, Renwick WE, Hogan C, Haeusler M, Tuckfield A, Tatoulis J: Recombinant activated factor VII: treating postoperative hemorrhage in cardiac surgery. Ann Thorac Surg 2006;81:875-879.

55 McCall P, Story DA, Karalapillai D: Audit of factor VIIa for bleeding resistant to conventional therapy following complex cardiac surgery. Can J Anaesth 2006;53:926-933. 